$\Rightarrow$ INFECTION

\section{ZIKA virus causes prostatitis}

New data show that Zika virus (ZIKV)

causes acute and chronic prostatitis in mice and macaques. These observations have implications for the potential long-term health effects of ZIKV infection in men.

First, the researchers investigated the effects of ZIKV infection on the prostates of mice. They created a model in which interferon signalling was blocked in male mice, sensitizing them to ZIKV infection. Mice were then inoculated with either Asian-American or African lineage ZIKV. The anterior, ventral, dorsal and lateral prostate lobes all contained high levels of ZIKV RNA 7 days post infection, which diminished at 21 days.

In mice infected with African-lineage ZIKV, histological analysis showed immune cell infiltration in the anterior, ventral, dorsal and lateral prostate lobes 14 days post infection. At 21 days after infection, the dorsal and lateral lobe tubules showed evidence of damage to the inner epithelial lining, hyperplasia of luminal epithelial cells and disruption of lumen architecture, with an increased density of nuclei, some of which were pyknotic. Cell death was apparent at 14 and 21 days post infection. At 90 days post infection, low levels of ZIKV RNA were present in the ventral lobe. Tubules with necrotic luminal cells were evident and mononuclear cell infiltration was persistent in the stroma. Disruption of luminal cell adhesions was also observed, as was the presence of proapoptotic cells.

In rhesus macaques (a more relevant, immunocompetent model of ZIKV infection than mice) infected with a Puerto Rican strain of ZIKV, prostates harvested 7 days after infection showed periglandular lymphocyte infiltration, formation of microabscesses and intraglandular neutrophils. At 35 days after infection, no ZIKV RNA was detected but periglandular inflammation was still evident.

These results show that ZIKV can cause acute and chronic prostatic inflammation, even after the virus is no longer present in the prostate.

Louise Stone

ORIGINAL ARTICLE Halabi, J. et al. Zika virus causes acute and chronic prostatitis in mice and macaques. J. Infect. Dis. https://doi.org/10.1093/infdis/jiz533 (2019)

RELATED ARTICLE Kurscheidt, F. A. et al. Persistence and clinical relevance of Zika virus in the male genital tract.

Nat. Rev. Urol. 16, 211-230 (2019)

\title{
New tools for Wilms tumour research
}

A new approach to 3D culturing of Wilms tumour cells in vitro has been described in Oncogene. Spheroids representing blastemal, stromal and epithelial Wilms tumour could be successfully grown, providing potential new preclinical models for investigating this disease.

Close inspection of the supernatant from culture of minced Wilms tumour samples revealed the presence of clusters of nonadherent cells. Culture of these cells in low-attachment plates plus rotary shaking and addition of a ROCK inhibitor to prevent anoikis resulted in five long-living $3 \mathrm{D}$ spheroid strains of different Wilms tumour histological subtypes. These spheroids could be passaged using mechanical disruption every 1-2 weeks and cultured for $>3$ years. They could be cryopreserved and revived with a cell viability rate of $60-80 \%$.

Characterization of the spheroids showed that they had the same allele loss pattern, genetic driver mutations, copy number variation and histological appearance as their respective parental tumours. Histological analysis showed that three spheroid strains comprised mostly blastemal cells, one was primarily epithelial in origin and the other contained mainly immature stromal cells.

Spheroids of each type could be successfully genetically manipulated, but stable transduction was not possible for blastemal-type spheroids. Furthermore, spheroids could be successfully xenografted into mice and, in vitro, spheroid cultures derived from xenografts had similar characteristics to the original tumour.

These spheroid cultures can be propagated long term using a simple, low-cost method. They maintain characteristics of the original tumour and can be genetically manipulated and xenografted to enable in vivo study, providing much-needed tools for preclinical investigation of Wilms tumour.

Louise Stone

ORIGINAL ARTICLE Wegert, J. et al. High-risk blastemal Wilms tumor can be modeled by $3 \mathrm{D}$ spheroid cultures in vitro. Oncogene https://doi.org/10.1038/s41388-019-1027-8 (2019)

\section{PROSTATE CANCER}

\section{Clinical decision-making with AR-V7}

A new study in European Urology shows that knowledge of the androgen receptor variant 7 protein (AR-V7) status of circulating tumour cells (CTCs) when making therapy decisions at the point of starting the second or greater line of therapy would prolong survival in men with progressing metastatic castration-resistant prostate cancer (mCRPC).

Men with mCRPC usually initially receive a next-generation AR signalling inhibitor (ARSI), but the best treatment after ARSI failure (taxane-based chemotherapy or a further ARSI) remains unclear. Two previous cohort analyses showed that men who had nuclear-localized AR-V7 in CTCs before starting second-line therapy had more favourable overall survival when receiving a taxane than when receiving an ARSI. In this new study, Graf et al. used those data with an extended follow-up period to investigate drivers of treatment choice when AR-V7 status is unknown and to estimate survival had the status been available to show clinical utility of an AR-V7 biomarker test. The analysis showed that the most influential drivers for physicians choosing a taxane were the number of previous systemic mCRPC therapies, liver metastases, PSA level and ARSI as the preceding treatment. Men who were assigned a taxane had more aggressive disease, more previous therapies and a higher risk of death than those assigned an ARSI.

Men assigned an ARSI had longer overall survival than those assigned a taxane (median 17.4 and 13.0 months; $P=0.00021$ ), but when adjusting for physician choice propensity no difference was seen. Notably, taking into account $A R-V 7$ status, $A R-V 7^{+}$men had longer survival when receiving a taxane than when receiving an ARSI (median 9.8 and 5.7 months; $P=0.041$ ), and $A R-V 7^{-}$men had, overall, longer survival when receiving an ARSI than when receiving a taxane $(P=0.033)$. Taken together, the analysis shows that survival would have been longer if data on nuclear AR-V7 status of CTCs had been available to guide treatment choice.

Clemens Thoma

ORIGINAL ARTICLE Graf, R. P. et al. Clinical utility of the nuclear-localized AR-V7 biomarker in circulating tumor cells in improving physician treatment choice in castrationresistant prostate cancer. Eur. Urol. https://doi.org/10.1016/ j.eururo.2019.08.020 (2019) 